\title{
Gravitational-wave signatures in successful vs. failed core-collapse supernovae
}

Kei Kotake*

Division of Theoretical Astronomy, National Astronomical Observatory of Japan, 2-21-1,

Osawa, Mitaka, Tokyo, 181-8588, Japan

E-mail: kkotake@th.nao.ac.jp

In this contribution, we discuss possible gravitational-wave (GW) signatures emitted from corecollapse supernovae that do or do not produce explosions. For the former case, we study properties of GWs based on three-dimensional (3D) supernova simulations, which demonstrate the neutrinodriven explosions aided by the standing accretion shock instability (SASI). By taking into account the effects of stellar rotation, we find that the gravitational waveforms from neutrinos in models that include rotation exhibit a common feature otherwise they vary much more stochastically in the absence of rotation. We point out that a recently proposed future space interferometers like Fabry-Perot type DECIGO would permit the detection of these signals for a Galactic supernova. For the black-hole forming supernovae, we study the GW emission based on a long-term special relativistic magnetohydrodynamic simulation in the light of collapsar model of long-duration gamma-ray bursts (GRBs). We find that the GWs from anisotropic neutrino emission illuminated by accretion disk become as high as the GWs contributed from matter motions of accreting material. These signals, possibly visible to the DECIGO-class detectors for a hundred Megaparsec distance scales, may give us an important probe into the central engines of GRBs.

25th Texas Symposium on Relativistic Astrophysics - TEXAS 2010

December 06-10, 2010

Heidelberg, Germany

* Speaker. 


\section{Introduction}

The successful detection of neutrinos from SN1987A paved the way for Neutrino Astronomy, an alternative to conventional astronomy by electromagnetic waves. Core-collapse supernovae are now expected to be opening yet another astronomy, Gravitational-Wave Astronomy. Currently long-baseline laser interferometers such as LIGO, VIRGO, GEO600, and TAMA300 are operational (e.g., [1] for a recent review). For these detectors, core-collapse supernovae have been proposed as one of the most plausible sources of gravitational waves (GWs) (see, for example, [2, 3] for recent reviews).

Traditionally, most of the theoretical predictions of GWs have focused on the bounce signals (e.g., see [月, 5, 2, 3] and references therein). However recent stellar evolution calculations suggest that rapid rotation assumed in most of the previous studies is not canonical for progenitors with neutron star formations [ 6 ]. Besides the rapid rotation of the core, convective matter motions and anisotropic neutrino emission in the much later postbounce phase are expected to be the primary GW sources with comparable amplitudes to the bounce signals. Thus far, various physical ingredients for producing asphericities and the resulting GWs in the postbounce phase have been studied, such as the roles of pre-collapse density inhomogeneities [7, 8, 9], moderate rotation of the iron core [10], nonaxisymmetric rotational instabilities (e.g., [11]), g-modes pulsations [12] of protoneutron stars (PNSs), and the standing accretion shock instability (SASI) (e.g., [13, 14, 15]).

However, most of them have been based on two-dimensional (2D) simulations that assume axisymmetry. Then, the growth of SASI (e.g., [16, 17, 18, 19, 20]) and the large-scale convection, both of which are now considered to generically develop in the postbounce phase and to help the neutrino-driven explosions [21, 22], develop along the symmetry axis preferentially, thus suppressing the anisotropies in explosions. There are several three-dimensional (3D) studies so far [8, 9], however the GW emission from these models have been poorly understood.

By performing 3D simulations that demonstrate the neutrino-driven core-collapse supernovae aided by SASI, we pointed out in our previous study [23] that the gravitational waveforms vary much more stochastically in 3D than 2D because the explosion anisotropies depend sensitively on the growth of the SASI which develops chaotically in all directions. In the former half of this contribution, we study the effects of rotation on the stochastic nature of the GW signals (section 2). In the latter half, we explore the GW signature emitted from the failing core-collapse supernovae in the light of collapsar simulations of long-duration GRBs (section 3).

\section{GWs from 3D exploding supernovae with rotation}

Figure 1 shows the gravitational waveforms for a typical 3D model without (top panels) and with rotation (bottom panels). For the rotating model, we give a uniform rotation on the flow advecting from the outer boundary of the iron core as in [24], whose specific angular momentum is assumed to agree with recent stellar evolution models [6]. Comparing these panels, one can clearly see the sudden rise in the GW amplitude from neutrinos seen from the equator after around 500 ms only for the model that includes rotation (bottom right panel). By changing the initial angular momentum as well as the input neutrino luminosities from the protoneutron star, we computed 

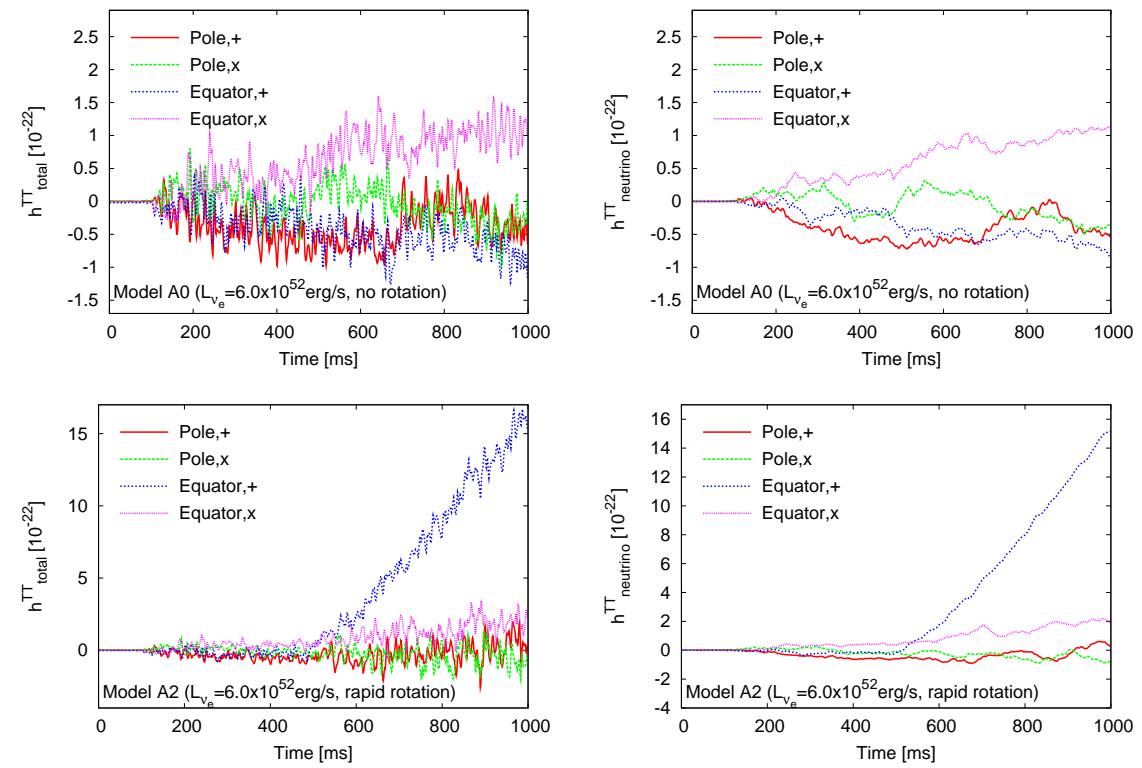

Figure 1: Gravitational waveforms from the sum of neutrinos and matter motions (left) and only from neutrinos (right) for models without (top panels), and with rotation (bottom panels). The time is measured from the epoch when the neutrino luminosity is injected from the surface of the neutrino sphere. For models presented here, the rotational flow is adjusted to advect to the PNS surface at around $t=400 \mathrm{~ms}$. The supernova is assumed to be located at the distance of $10 \mathrm{kpc}$. We performed the ray-tracing calculation to estimate correctly the GWs generated by anisotropic neutrino emission [14].

fifteen 3D models. The GW features mentioned above are found to be common to the other rotating models.

Figure 1 illustrates a typical snapshot of the flow fields for the rotating model when the spiral SASI modes have already entered the non-linear regime, seen from the pole (right panel) or from the equator (left panel), respectively. From the left panel, one may guess the presence of the sloshing modes that happen to develop along the rotational axis (z-axis) at this epoch. It should be emphasized that the dominance of $h_{v,+}^{\text {equ }}$ observed in the current 3D simulations have nothing to do with the one found in our previous 2D studies [14]. Free from the 2D axis effects, the major axis of the SASI changes stochastically with time, and the flow patters behind the standing shock simultaneously change in every direction. As a result, the sloshing modes can make only a small contribution to the GW emission. The remaining possibility is that the spiral flows seen in the right panel should be a key importance to understand the GW feature mentioned above. In fact, by analyzing the matter distribution on the equatorial plane, we find that the compression of matter is more enhanced in the vicinity of the equatorial plane due to the growth of the spiral SASI modes, leading to the formation of the spiral flows circulating around the spin axis with higher temperatures. As a result, the neutrino emission seen parallel to the spin axis becomes higher than the ones seen from the other direction. Remembering again that the lateral-angle $(\theta)$ dependent function of the GW formulae (e.g., in equation (9) in [14]) is positive near the north and south polar caps, the dominance of the polar neutrino luminosities leads to make the positively growing feature 
of $h_{v,+}^{\text {equ }}$ in Figure 1. By performing the spectral analysis of the gravitational waveforms (Figure 3), it can be readily seen that it is very difficult to detect these neutrino-originated GW signatures with slower temporal evolution $(\sim>O(10) \mathrm{ms})$ by ground-based detectors whose sensitivity is limited mainly by the seismic noises at such lower frequencies. However these signals may be detectable by the recently proposed future space interferometers like Fabry-Perot type DECIGO [25] (black line in Figure 3). Contributed by the neutrino GWs in the lower frequency domains, the total GW spectrum tends to become rather flat over a broad frequency range below $\sim 100 \mathrm{~Hz}$. These GW features obtained in the context of the SASI-aided neutrino-driven mechanism are different from the ones expected in the other candidate supernova mechanism, such as the MHD mechanism (e.g., [26]) and the acoustic mechanism [12]. Therefore the detection of such signals is expected to provide an important probe into the long-veiled explosion mechanism.
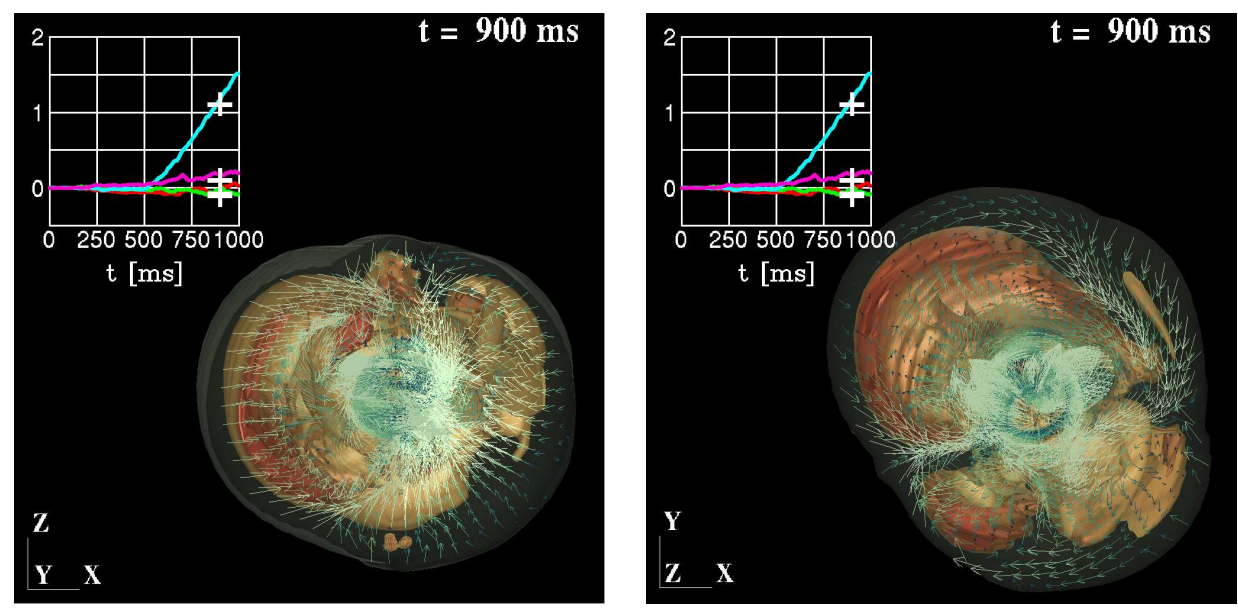

Figure 2: Partial cutaway of the entropy isosurfaces and the velocity vectors on the cutting plane for the rotating model. Left and right panels are for the equatorial and polar observer, respectively. The insets show the gravitational waveforms with ' + ' on each curves representing the time of the snapshot. Note that the colors of the curves are taken to be the same as the top panel of Figure 1.

\section{GWs from collapsar evolution}

To explore the GW emission from black-hole (BH) forming supernovae, we estimate the GW signals based on our long-term collapsar simulation in special relativistic magnetohydrodynamics (e.g., [30]). Figure 4 depicts a snapshot taken from our collapsar simulation (for model J0.8 in [30]), showing a clear accretion-disk and BH system with the polar funnel regions along the spin axis of the disk. The dynamically rotating accretion disk is the primary source of the GWs from matter motions, while the anisotropy of neutrinos coming out from the surface of the accretion disk (white line) gives rise to the neutrino-originated GWs. In fact, the neutrino luminosity seen from the spin axis (left panel in Figure 5) becomes higher than the one seen from the equatorial direction (right panel), because the cross section of the pan-cake like accretion disk seen from the spin axis becomes larger compared to the one seen from the horizontal direction. To estimate the neutrino anisotropy in curved space, we performed the general relativistic ray-tracing calculation 


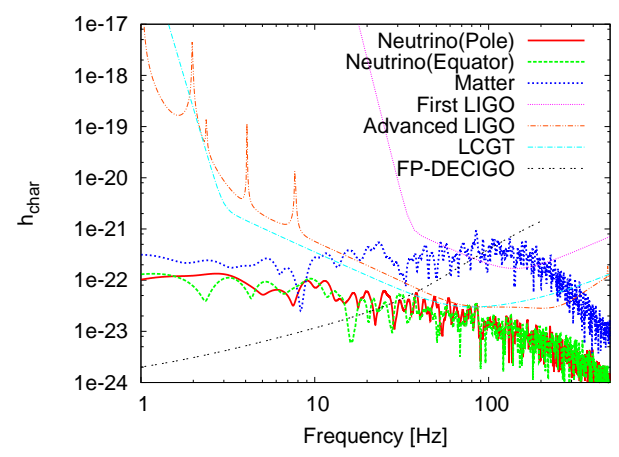

Figure 3: Spectral distributions of GWs from matter motions ("Matter") and neutrino emission ("Neutrino") seen from the pole or the equator for the rotating model with the expected detection limits of TAMA300 [27], first LIGO and advanced LIGO [28], Large-scale Cryogenic Gravitational wave Telescope (LCGT) [29] and Fabry-Perot type DECIGO [25]. The distance to the supernova is assumed to be $10 \mathrm{kpc}$. Note that for the matter signal, the + mode seen from the polar direction is plotted.

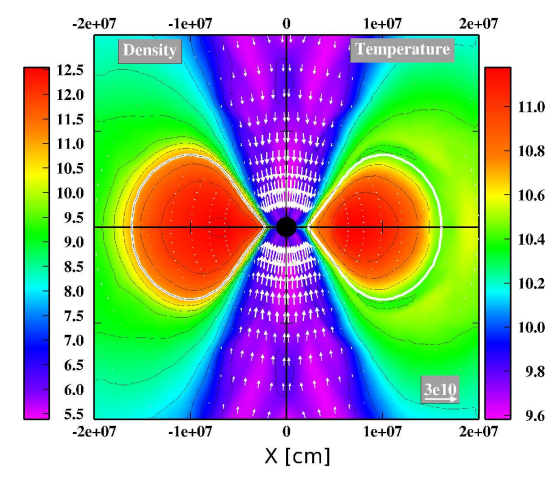

Figure 4: A snapshot for our collapsar simulation (at $9.1 \mathrm{~s}$ after the onset of gravitational collapse for model J0.8 when the accretion disk is in a stationary state (see [30] for more detail). The logarithmic density (in, left-half) and temperature (in $K$, right-half) are shown. The white solid line denotes the area where the density is equal to $10^{11}$, representing the surface of the accretion disk. The central black circle $\left(\approx 4 M_{\odot}\right)$ represents the inner boundary of our computations.

[31]. The left panel in Figure 6 shows the contribution to the total GW amplitudes (red line) from neutrinos (light blue line) and from matter motions (green line + purple line), respectively. As well known, the sign of the GW amplitudes from oblately deforming object is negative. This is the reason of the negative growth of the matter GWs. On the other hand, the positively growing feature of the neutrino GWs is due to the excess of neutrino emission parallel to the rotational axis. This argument is essentially the same as the one in the previous section. From the right panel, it can be seen that the DECIGO-class detectors in the next generation would permit the detection of these signals, depending on the initial angular momentum of precollapse progenitor (indicated by J0.6 and J0,8 in the panel (see, [30] for more details), for a $100 \mathrm{Mpc}$ distance scale. If the longduration GRBs are originated from the so-called collapsar, as it is receiving quite some interest for more than decade (e.g., [32]), the detection of these signals may provide us an important clue to 

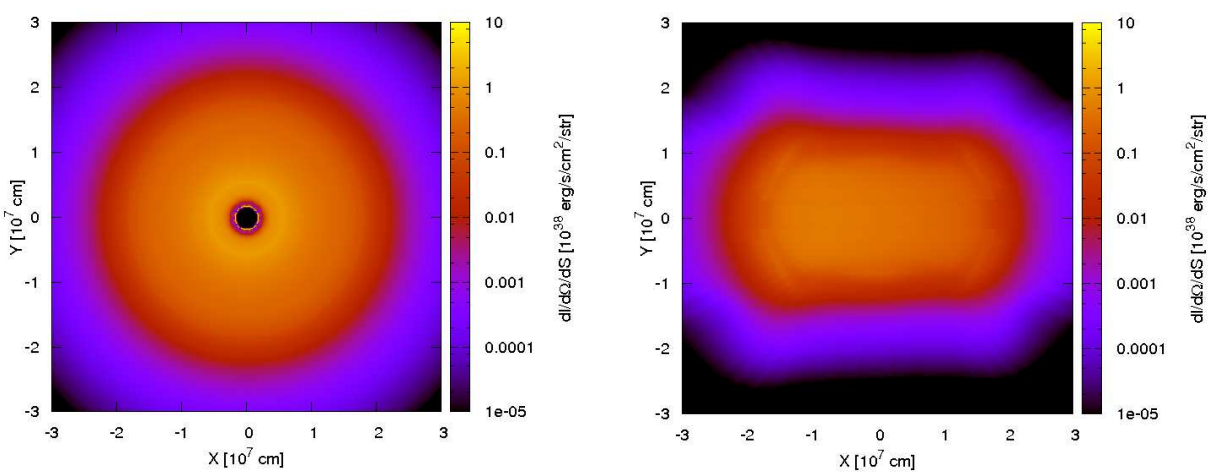

Figure 5: Top (left panel) and side view (right panel) of the neutrino energy fluxes irradiating from the accretion disk (for model J0.8). The central black hole $\left(\sim 3 M_{\odot}\right)$ is assumed to be a maximally rotating with $a=0.99$, where $a$ is the Kerr parameter.

understand their long-veiled central engines.

It is gratefully acknowledged that the results presented in this article are the outcome of the collaborations with W. Iwakami Nakano, N. Ohnishi, S. Harikae, and T. Takiwaki. Numerical computations were in part carried on XT4 and general common use computer system at the center for Computational Astrophysics, CfCA, the National Astronomical Observatory of Japan. This study was supported in part by the Grants-in-Aid for the Scientific Research from the Ministry of Education, Science and Culture of Japan (Nos. 19540309 and 20740150).
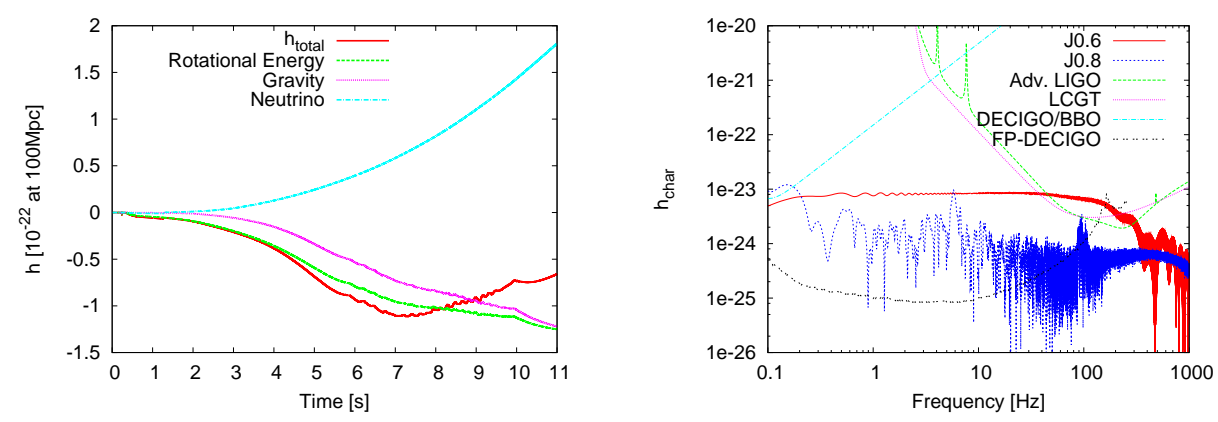

Figure 6: The left panel is the gravitational waveform for model $\mathrm{J} 0.8$, in which the contribution to the total amplitudes (red line) comes from the GWs generated by anisotropic neutrino emission (light blue line) and from matter motions (green line + purple line). The right panel shows the gravitational spectrum for some representative collapsar models (models J0.6 and J0.8) with the expected detection limits of LCGT, BBO, LCGT [29] and Fabry-Perot type DECIGO [25], respectively (see text for more detail). The distance to the source is taken to be $100 \mathrm{Mpc}$.

\section{References}

[1]Hough, J., \& Rowan, S. 2005, Journal of Optics A: Pure and Applied Optics, 7, 257 
[2]Kotake, K., Sato, K., \& Takahashi, K. 2006, Reports of Progress in Physics, 69, 971

[3]Ott, C. D 2009, Classical and Quantum Gravity, 26, 063001

[4]Kotake, K., Yamada, S., \& Sato, K. 2003, Phys. Rev. D, 68, 044023

[5]Kotake, K., Yamada, S., Sato, K., Sumiyoshi, K., Ono, H., \& Suzuki, H. 2004, Phys. Rev. D, 69, 124004

[6]Heger, A., Woosley, S. E., \& Spruit, H. C. 2005, Astrophys. J., 626, 350

[7]Burrows, A. \& Hayes, J. 1996, Physical Review Letters, 76, 352

[8]Müller, E., \& Janka, H.-T. 1997, A\&A, 317, 140

[9]Fryer, C. L., Holz, D. E., \& Hughes, S. A. 2004, ApJ, 609, 288

[10]Müller, E., Rampp, M., Buras, R., Janka, H.-T., \& Shoemaker, D. H. 2004, Astrophys. J., 603, 221

[11]Andersson, N., Ferrari, V., Jones, D. I., Kokkotas, K. D., Krishnan, B., Read, J. S., Rezzolla, L., \& Zink, B. 2010, General Relativity and Gravitation, 156

[12]Ott, C. D., Burrows, A., Dessart, L., \& Livne, E. 2006, Physical Review Letters, 96, 201102

[13]Kotake, K., Ohnishi, N., \& Yamada, S. 2007, Astrophys. J., 655, 406

[14]Kotake, K., Iwakami, W., Ohnishi, N., \& Yamada, S. 2009, Astrophys. J, 704, 951

[15]Marek, A., Janka, H.-T., Müller, E. 2009, A\&A, 496, 475

[16]Blondin, J. M., Mezzacappa, A., \& DeMarino, C. 2003, Astrophys. J., 584, 971

[17]Scheck, L., Plewa, T., Janka, H.-T., Kifonidis, K., Müller, E. 2004, Physical Review Letters, 92, 011103

[18]Ohnishi, N., Kotake, K., \& Yamada, S. 2006, Astrophys. J., 641, 1018

[19]Foglizzo, T., Galletti, P., Scheck, L., \& Janka, H.-T. 2007, Astrophys. J., 654, 1006

[20]Iwakami, W., Kotake, K., Ohnishi, N., Yamada, S., \& Sawada, K. 2008, Astrophys. J., 678, 1207

[21]Bethe, H. A. 1990, Reviews of Modern Physics, 62, 801

[22]Marek, A., \& Janka, H.-T. 2009, Astrophys. J., 694, 664

[23]Kotake, K., Iwakami, W., Ohnishi, N., \& Yamada, S. 2009, Astrophys. J., 697, L133

[24]Iwakami, W., Kotake, K., Ohnishi, N., Yamada, S., \& Sawada, K. 2009, Astrophys. J., 700, 232

[25]Kawamura, S., et al. 2006, Classical and Quantum Gravity, 23, 125

[26]Takiwaki, T., \& Kotake, K. 2010, arXiv:1004.2896

[27]Ando, M., \& the TAMA Collaboration 2005, Classical and Quantum Gravity, 22, 881

[28]Abbott, B., et al. 2005, Phys. Rev. D, 72, 122004

[29]Kuroda, K., \& LCGT Collaboration 2010, Classical and Quantum Gravity, 27, 084004

[30]Harikae, S., Takiwaki, T., \& Kotake, K. 2009, Astrophys. J., 704, 354

[31]Harikae, S., Kotake, K., Takiwaki, T., \& Sekiguchi, Y.-i. 2010, Astrophys. J,, 720, 614

[32]MacFadyen, A. I., \& Woosley, S. E. 1999, Astrophys. J., 524, 262 